Internat. J. Math. \& Math. Sci.

Vol. 23, No. 3 (2000) 169-174

S0161171200000636

(C) Hindawi Publishing Corp.

\title{
A GENERALIZATION OF A THEOREM OF FAITH AND MENAL AND APPLICATIONS
}

\author{
KENTARO TSUDA
}

(Received 19 December 1997 and in revised form 10 March 1998)

\begin{abstract}
In 1995, Faith and Menal have established the $V$-ring theorem which gives a characterization of a $V$-ring. In this paper, we generalize this theorem to $V$-modules and consider some applications for Noetherian self-cogenerators.
\end{abstract}

Keywords and phrases. $V$-module, $V$-ring, Johns ring, strongly Johns ring.

2000 Mathematics Subject Classification. Primary 16E50.

1. Preliminaries. Throughout this paper, $R$ denotes an associative ring with identity and all modules considered are unitary right $R$-modules. Homomorphisms are written on the side opposite to that of scalars. For any module $M$, the sum of all simple submodules of $M$ is called a socle of $M$ and is denoted by $\operatorname{Soc}(M)$. Dually, the intersection of all maximal submodules of $M$ is called a radical of $M$ and is denoted by $\operatorname{Rad}(M)$. $(R)_{n}$ denotes the $n \times n$ matrix ring over $R$. Let $M$ be a module. An $M$-generated module is a module which is isomorphic to a factor module of $M^{(I)}$ for some index set $I$. We denote by $\sigma[M]$ the full subcategory of Mod-R whose objects are all submodules of $M$-generated modules, and by $E_{M}(N)$ the $M$-injective hull of a module $N$ in $\sigma[M]$ which is the trace of $M$ in $E(M)$, where $E(M)$ indicates the injective hull of $M$, that is $E_{M}(N)=\sum\left\{f(M): f \in \operatorname{Hom}_{R}(M, E(N))\right\}$ in $\sigma[M]$ (see Wisbauer [9, $17.9,(2)])$. A module $M$ is called a $V$-module if every proper submodule of $M$ is an intersection of maximal submodules of $M$ or, equivalently, if every simple module (in $\sigma[M]$ or Mod- $R$ ) is $M$-injective (see, e.g., Wisbauer [9, 23.1]). A ring $R$ is called a right $V$-ring if $R$ is a $V$-module when considered as a right module over itself, i.e., every simple module is injective. For notation, definitions and, familiar results concerning the ring theory we mainly follow Anderson and Fuller [2] and Wisbauer [9].

2. A generalization of a theorem of Faith and Menal. Let $M, E$ be modules, and $M_{E}^{*}=\operatorname{Hom}_{R}(M, E)$. For each subset $Z$ of $M_{E}^{*}$ and each subset $X$ of $M$, the right annihilator in $M$ is denoted by $r_{M}(Z)$, and the left annihilator in $M_{E}^{*}$ is denoted by $\ell_{M_{E}^{*}}(X)$, that is,

$$
r_{M}(Z)=\{m \in M: Z m=0\}, \quad \ell_{M_{E}^{*}}(X)=\left\{f \in M_{E}^{*}: f X=0\right\} .
$$

In [6], Faith and Menal showed that a ring $R$ is a right $V$-ring if and only if there exists a semisimple module $W$ such that $I=r_{R} \ell_{W}(I)$ for every right ideal $I$ of $R$. In this case, we say that $W$ satisfies the double annihilator condition (d.a.c.) with respect to right ideals. This characterization of a $V$-ring by the existence of a duality between the right 
ideals via annihilation and submodules of a semisimple module is called the $V$-ring theorem. A ring $R$ is called a right Johns ring if $R$ is right Noetherian and satisfies that any right ideal is a right annihilator ideal. It is known that a right Johns ring is not right Artinian (see Faith and Menal [4]). If a ring $R$ is right Johns, then $\tilde{I}=r_{R / J(R)} \ell_{\operatorname{Soc}(R)}(\tilde{I})$ for any right ideal $\tilde{I}$ of $R / J(R)$, that is $R / J(R)$ is a right $V$-ring by the $V$-ring theorem, where $J(R)$ denotes the Jacobson radical of $R$ (see Faith and Menal [6]). We begin with the following theorem.

THEOREM 2.1. Let $M$ be a module. Then the following are equivalent:

(1) $M$ is a $V$-module;

(2) there exists a semisimple module $W$ satisfying $N=r_{L} \ell_{L_{W}^{*}}(N)$ for any module $L$ in Mod- $R$ and any submodule $N$ of $L$ such that $L / N$ is in $\sigma[M]$;

(3) there exists a semisimple module $W^{\prime}$ in $\sigma[M]$ satisfying $N=r_{L} \ell_{L_{W^{\prime}}^{*}}(N)$ for any module $L$ in $\sigma[M]$ and any submodule $N$ of $L$.

Proof. $(1) \Rightarrow(2),(1) \Rightarrow(3)$. Let $\left\{S_{i}\right\}_{i \in \Omega}$ be an irredundant set of representatives of the simple modules in $\sigma[M]$. Then, $\bigoplus_{i \in \Omega} E_{M}\left(S_{i}\right)$ is the minimal $M$-injective cogenerator of $\sigma[M]$ (see Wisbauer [9, p. 143]).

Since $M$ is a $V$-module, $E_{M}\left(S_{i}\right)=S_{i}$ for each $i \in \Omega$ and, hence, $\bigoplus_{i \in \Omega} S_{i}$ is a semisimple cogenerator of $\sigma[M]$. Hence, $\bigoplus_{i \in \Omega} S_{i}$ cogenerates $L / N$ for any module $L$ and any submodule $N \subseteq L$ such that $L / N$ in $\sigma[M]$. By Albu and Năstăsescu [1, Prop. 3.5], $\bigoplus_{i \in \Omega} S_{i}$ cogenerates the factor module $L / N$ if and only if $N=r_{L} \ell_{L_{\oplus_{i \in \Omega}^{*}}^{*} S_{i}}(N)$. Now, the proof of $(1) \Rightarrow(2)$ is clear.

Since $\bigoplus_{i \in \Omega} S_{i}$ is in $\sigma[M]$ and, for any module $L$ in $\sigma[M]$, each factor module of $L$ belongs to $\sigma[M]$, the implication $(1) \Rightarrow(3)$ also follows from the proof above.

$(2) \Rightarrow(1),(3) \Rightarrow(1)$. For a semisimple module $W$ satisfying condition (2), since each factor module of $M$ belongs to $\sigma[M]$, we see that $N=r_{M} \ell_{M_{W}^{*}}(N)$ holds for any submodule $N$ of $M$. Hence, $M / N \rightarrow W^{\ell_{M_{W}^{*}}^{*}}, m+N \mapsto(f(m))_{f \in \ell_{M_{W}^{*}(N)}}$ is an $R$-monomorphism. This readily implies that $\operatorname{Rad}(M / N)=0$. Hence, $N$ is an intersection of maximal submodules of $M$. Thus, $M$ is a $V$-module. For a semisimple module $W^{\prime}$ satisfying condition (3), it also follows from the same argument above that $M$ is a $V$-module.

COROLLARY 2.2. Let $M$ be a module. Then the following statements are equivalent:

(1) $M$ is a $V$-module;

(2) there exists a semisimple module $W$ satisfying $I=r_{R} \ell_{W}(I)$ for any right ideal $I$ of $R$ such that $R / I$ is in $\sigma[M]$;

(3) there exists a semisimple module $W^{\prime}$ in $\sigma[M]$ satisfying $N=r_{M} \ell_{M_{W^{\prime}}^{*}}(N)$ for any submodule $N$ of $M$.

In this case, $W$ and $W^{\prime}$ cogenerate any module in $\sigma[M]$.

Proof. $(1) \Rightarrow(2),(1) \Rightarrow(3)$. These are obvious by Theorem 2.1.

$(3) \Rightarrow(1)$. Follows immediately from the same argument of $(3) \Rightarrow(1)$ in the proof of Theorem 2.1.

(2) $\Rightarrow(1)$. Let $S$ be any simple module in $\sigma[M]$. To show that $S$ is $M$-injective, we need to show that $S$ is $N$-injective for every cyclic submodule $N$ of $M$ by Wisbauer [9, 16.3, (b)]. So, let $N$ be a cyclic submodule of $M$ and let $f$ be a nonzero $R$-homomorphism 
from a submodule $N^{\prime}$ of $N$ to $S$. Since $N$ is cyclic, $N \cong R / I$ for some right ideal $I$ of $R$ and, hence, $N^{\prime} \cong L / I$ for some right ideal $L$ of $R$. Therefore, $\operatorname{Ker}(f) \cong L^{\prime} / I$ for some right ideal $L^{\prime} \subset L$ of $R$. Since $N$, $\operatorname{Ker}(f)$ are in $\sigma[M]$ and since $\sigma[M]$ is closed under cokernels, $R / L^{\prime}$ is in $\sigma[M]$. The hypothesis implies that $L^{\prime}=r_{R} \ell_{W}\left(L^{\prime}\right)$. By [1, Prop. 3.5], there is an exact sequence $0 \rightarrow R / L^{\prime} \rightarrow W^{Y}$ for some set $Y$. This readily implies that $\operatorname{Rad}\left(R / L^{\prime}\right)=0$. Then since $L^{\prime}$ is an intersection of maximal right ideals, there is a maximal right ideal $K$ of $R$ such that $K \supseteq L^{\prime}$ but $K \nsupseteq L$. Since $N^{\prime} / \operatorname{Ker}(f) \cong L / L^{\prime}$ is simple, it follows that $L \cap K=L^{\prime}$. Then $R / I / K / I \cong R / K=(L+K) / K \cong(L / L \cap K)=$ $L / L^{\prime} \cong N^{\prime} / \operatorname{Ker}(f) \cong S$ and, therefore, $f$ can be extended to an $\bar{f}$ in $\operatorname{Hom}_{R}(N, S)$. Hence, $S$ is $N$-injective and $M$ is a $V$-module.

Finally, we show that a semisimple module $W$ satisfying condition (2) and a semisimple module $W^{\prime}$ satisfying condition (3) cogenerate any module in $\sigma[M]$. For any maximal right ideal $I$ with $R / I$ in $\sigma[M]$, we observe that $I=r_{R} \ell_{W}(I)$ holds. Thus, it follows, by almost same argument in the proof of the corollary in Faith and Menal [6], that $W$ satisfying condition (2) cogenerates any module in $\sigma[M]$. Next, since $E_{M}(S)=S$ for any simple module $S$ in $\sigma[M], f(M)=S$ for some $f \in \operatorname{Hom}_{R}(M, S)$ and, hence, $M / \operatorname{Ker}(f) \cong S$. Then since $W^{\prime}$ satisfies the d.a.c. with respect to the submodules of $M, \operatorname{Ker}(f)=r_{M} \ell_{M_{W^{\prime}}^{*}}(\operatorname{Ker}(f))$. Since $\operatorname{Ker}(f)$ is maximal, $\operatorname{Ker}(f)=r_{M}(g)=\operatorname{Ker}(g)$ for some $g \in M_{W^{\prime}}^{*}$. Therefore, $W^{\prime}$ contains a copy of $S$. This implies that $W^{\prime}$ satisfying condition (3) cogenerates any module in $\sigma[M]$.

REMARK 2.3. Let $M$ be a module. If there exists a semisimple module $W$, which need not be in $\sigma[M]$, such that $W$ satisfies the d.a.c. with respect to any submodule of $M$, then it is easy to deduce from the argument of the proof of $(2) \Rightarrow(1)$ and $(3) \Rightarrow(1)$ in Theorem 2.1 that $M$ is a $V$-module.

Proposition 2.4. Let $M$ be a module. If $M$ contains a copy of each simple factor module of $M$, then the following statements are equivalent:

(1) $M / \operatorname{Rad}(M)$ is a $V$-module;

(2) $\operatorname{Soc}(M)$ cogenerates any module in $\sigma[M / \operatorname{Rad}(M)]$;

(3) $\tilde{I}=r_{R / J(R)} \ell_{\operatorname{Soc}(M)}(\tilde{I})$ for any right ideal $\tilde{I}$ of $R / J(R)$ such that $(R / J(R)) / \tilde{I}$ is in $\bar{\sigma}[M / \operatorname{Rad}(M)]=\sigma[M / \operatorname{Rad}(M)] \cap(\operatorname{Mod}-R / J(R))$.

Proof. $(1) \Rightarrow(2)$. Let $\{S\}_{i \in \Omega}$ be an irredundant set of representatives of the simple $R$-modules in $\sigma[M / \operatorname{Rad}(M)]$. Since $M / \operatorname{Rad}(M)$ is a $V$-module, by Wisbauer [9, p. 143], we know that $\bigoplus_{i \in \Omega} S_{i}$ cogenerates any module in $\sigma[M / \operatorname{Rad}(M)]$. So, it suffices to show that $\operatorname{Soc}(M)$ contains a copy of $S_{i}$ for each $i \in \Omega$. Since $E_{M / \operatorname{Rad}(M)}\left(S_{i}\right)=$ $S_{i}, f(M / \operatorname{Rad}(M))=S_{i}$ for some $f \in \operatorname{Hom}_{R}\left(M / \operatorname{Rad}(M), S_{i}\right)$. Clearly, $S_{i}$ is a simple homomorphic image of $M$. Thus, by hypothesis, there exists an exact sequence $0 \rightarrow S_{i} \rightarrow$ $\operatorname{Soc}(M)$. Obviously, it follows that $\operatorname{Soc}(M)$ cogenerates any module in $\sigma[M / \operatorname{Rad}(M)]$.

$(2) \Rightarrow(3)$. We note that any module in $\bar{\sigma}[M / \operatorname{Rad}(M)]$ belongs to $\sigma[M / \operatorname{Rad}(M)]$. Since $\operatorname{Soc}(M)$ cogenerates any module in $\bar{\sigma}[M / \operatorname{Rad}(M)]$, again by virtue of [1, Prop. 3.5], we have $\tilde{I}=r_{R / J(R)} \ell_{\text {Soc }}(M)(\tilde{I})$ for every right ideal $\tilde{I}$ of $R / J(R)$ such that $R / J(R) / \tilde{I}$ in $\bar{\sigma}[M / \operatorname{Rad}(M)]$.

(3) $\Rightarrow(1)$. Note that $M / \operatorname{Rad}(M)$ is a $V$-module as a right $R / J(R)$-module if and only if $M / \operatorname{Rad}(M)$ is a $V$-module as a right $R$-module. Since $M / \operatorname{Rad}(M)_{R / J(R)}$ is a $V$-module by Corollary 2.2, $M / \operatorname{Rad}(M)_{R}$ is a $V$-module. 
Recall that a ring $R$ is a right Kasch ring if any simple right $R$-module is isomorphic to a minimal right ideal of $R$. Since a ring $R$ is right Kasch if and only if every maximal right ideal of $R$ is a right annihilator ideal (see, e.g., Faith [3, p. 37]), we observe that a right Johns ring is right Kasch.

COROLLARY 2.5. If a ring $R$ is right Kasch, then the following statements are equivalent:

(1) $R / J(R)$ is a right $V$-ring;

(2) $\operatorname{Soc}(R)$ cogenerates any module in $\operatorname{Mod}-R / J(R)$;

(3) $\tilde{I}=r_{R / J(R)} \ell_{\operatorname{Soc}(R)}(\tilde{I})$ for every right ideal $\tilde{I}$ of $R / J(R)$.

3. Applications. A module $M$ is called a self-generator if $M$ generates every submodule of $M$. Dually, a module $M$ is called a self-cogenerator if $M$ cogenerates every factor module of $M$. By Albu and Năstăseacu [1, Prop. 3.5], $M$ is a self-cogenerator if and only if $N=r_{M} \ell_{\Lambda}(N)$ for any submodule $N$ of $M$, where $\Lambda=\operatorname{End}\left(M_{R}\right)$. In particular, $R_{R}$ is a self-cogenerator if and only if $I=r_{R} \ell_{R}(I)$ for any right ideal $I$ of $R$.

THEOREM 3.1. Let $M$ be a self-cogenerator and let $\Lambda=\operatorname{End}\left(M_{R}\right)$. If there exists a $(\Lambda, R)$-bimodule $W \subseteq \operatorname{Soc}\left(M_{R}\right)$ such that $M_{W}^{*}=\ell_{\Lambda}(X)$ for some subset $X$ of $M$, then $\bar{M}=M / r_{M}\left(M_{W}^{*}\right)$ is a $V$-module.

Proof. By virtue of Remark 2.3, we need to prove that $N / r_{M}\left(M_{W}^{*}\right)=r_{\bar{M}} \ell_{\bar{M}_{W}^{*}}(N /$ $r_{M}\left(M_{W}^{*}\right)$ ) for every submodule $N \supseteq r_{M}\left(M_{W}^{*}\right)$ of $M$. Applying the $W$-dual functor $\operatorname{Hom}_{R}(-, W)$ to the natural exact sequence $M \rightarrow \bar{M} \rightarrow 0$, we get that the dual sequence $0 \rightarrow \bar{M}_{W}^{*} \rightarrow M_{W}^{*}$ is exact. Since $\ell_{\Lambda} r_{M}\left(M_{W}^{*}\right)=\ell_{\Lambda} r_{M} \ell_{\Lambda}(X)=\ell_{\Lambda}(X)=M_{W}^{*}$ by hypothesis, we have $M_{W}^{*} \cong \bar{M}_{W}^{*}$ as an abelian group. Since $M$ is a self-cogenerator, there exists a subset $\left\{g_{i}\right\}_{i \in I} \subseteq \Lambda$ such that $N=r_{M}\left(\left\{g_{i}\right\}_{i \in I}\right)$. If we take the left annihilator in $\Lambda$ for $r_{M}\left(M_{W}^{*}\right) \subseteq N$, we have $\left\{g_{i}\right\}_{i \in I} \subseteq \ell_{\Lambda}(N) \subseteq \ell_{\Lambda} r_{M}\left(M_{W}^{*}\right)=M_{W}^{*}$. Since $M_{W}^{*} \cong \bar{M}_{W}^{*}$ by the natural way, so that $\left\{\bar{g}_{i}\right\}_{i \in I} \subseteq \bar{M}_{W}^{*}$ follows, where $\bar{g}_{i}: \bar{M} \rightarrow W$ denotes the $R$-homomorphism induced by $g_{i}$ for each $i \in I$. Thus, we obtain that $\left\{\bar{g}_{i}\right\}_{i \in I} \subseteq \ell_{\bar{M}_{W}^{*}}\left(N / r_{M}\left(M_{W}^{*}\right)\right)$. So, if we note that $r_{\bar{M}}\left(\left\{\bar{g}_{i}\right\}_{i \in I}\right)=r_{M}\left(\left\{g_{i}\right\}_{i \in I}\right) / r_{M}\left(M_{W}^{*}\right)$, then we have

$$
r_{\bar{M}} \ell_{\bar{M}_{W}^{*}}\left(\frac{N}{r_{M}\left(M_{W}^{*}\right)}\right) \subseteq r_{\bar{M}}\left(\left\{\bar{g}_{i}\right\}_{i \in I}\right)=\frac{r_{M}\left(\left\{g_{i}\right\}_{i \in I}\right)}{r_{M}\left(M_{W}^{*}\right)}=\frac{N}{r_{M}\left(M_{W}^{*}\right)}
$$

Since the reverse inclusion is easily verified, this completes the proof.

Observe that a right Johns ring is a trivial Noetherian self-cogenerator. Next, we consider a nontrivial module which is a Noetherian self-cogenerator. It is known that the class of right Johns rings is not Morita stable (see Faith and Menal [5, Rem. 3.7]). A ring $R$ is called a strongly right Johns ring if $(R)_{n}$ is right Johns for all positive integers $n$. However, it is not known if a strongly right Johns ring must be quasiFrobenius, equivalently, right Artinian (cf. Faith and Menal [5]). Using a right Johns ring and a strongly right Johns ring, we construct Noetherian self-cogenerators. Let $n>0, S=(R)_{n}$ and $P=R^{(n)}$. Consider the functor $H=\operatorname{Hom}_{R}(P,-): \operatorname{Mod}-R \rightarrow \operatorname{Mod}-S$. We note that the functor $H=\operatorname{Hom}_{R}(P,-): \operatorname{Mod}-R \rightarrow \operatorname{Mod}-S$ is an equivalence.

EXAMPLE 1. Suppose that $R$ is a strongly right Johns ring and consider $P=R^{(n)}$. Since $H(R)^{n} \cong H(P) \cong S$, every factor module of $P_{R}$ is cogenerated by $R$ if and only if 
every factor module of $S_{S}$ is cogenerated by $H(R)$ if and only if every factor module of $S_{S}$ is cogenerated by $S$. Thus, $P_{R}$ gives an example of a Noetherian self-cogenerator.

EXAMPLE 2. Suppose that $R$ is a right Johns ring and consider $P=R^{(n)}$ as a right $S$-module by the usual way. By Anderson and Fuller [2, Prop. 21.7], each submodule of $H(R)_{S}$ is of the form $\operatorname{Im} H(g)$ for some submodule $I$ of $R_{R}$ and the inclusion map $g: I \rightarrow R$. Since $R_{R}$ is a self-cogenerator, $I=r_{R} \ell_{R}(I)$ holds for any right ideal $I$ of $R$. By Kurata and Hashimoto [8, Lem. 1.19], we have $\operatorname{Im} H(g)=r_{H(R)} \ell_{R}(\operatorname{Im} H(g))$. Then, $H(R) / \operatorname{Im} H(g) \rightarrow H(R)^{\ell_{R}(\operatorname{Im} H(g))}, m+\operatorname{Im} H(g) \mapsto(r m)_{r \in \ell_{R}(\operatorname{Im} H(g))}$ is an $S$-monomorphism. Thus, $H(R)_{S}$ is a self-cogenerator. Since $P_{S} \cong H(R)_{S}$ is a natural isomorphism, $H(R)_{S}$ is a self-cogenerator if and only if $P_{S}$ is a self-cogenerator. Thus $P_{S}$ is a selfcogenerator. Since $S$ is right Noetherian, the finitely generated module $P_{S}$ is right Noetherian. Therefore, $P_{S}$ gives an example of a Noetherian self-cogenerator.

Proposition 3.2. If $M$ is a Noetherian projective self-cogenerator, then $\Lambda=\operatorname{End}\left(M_{R}\right)$ is a right Johns ring and $\operatorname{End}\left(M / \operatorname{Rad}(M)_{R}\right)$ is a right $V$-ring.

Proof. Suppose that $I$ is any finitely generated right ideal of $\Lambda$. Since $M$ is projective, $I=\operatorname{Hom}_{R}(M, I M)$ by Wisbauer $[9,18.4]$. Since $M$ is a self-cogenerator, there is some set $Y$ of $\Lambda$ such that $I M=r_{M}(Y)$. Now, it is straightforward to verify that

$$
\operatorname{Hom}_{R}\left(M, r_{M}(Y)\right)=r_{\Lambda}(Y) .
$$

This implies that $I$ is a right annihilator ideal. Since $M$ is Noetherian and projective, it follows from Albu and Năstăsescu [1, Prop. 4.12] that $\Lambda$ is right Noetherian. Hence, $\Lambda$ is a right Johns ring. Now, by Anderson and Fuller [2, Cor. 17.12], $\operatorname{End}\left(M / \operatorname{Rad}(M)_{R}\right) \cong$ $\Lambda / J(\Lambda)$. Since $\Lambda / J(\Lambda)$ is a right $V$-ring, $\operatorname{End}\left(M / \operatorname{Rad}(M)_{R}\right)$ is a right $V$-ring.

COROLLARY 3.3. Let $M$ be a Noetherian projective self-generator and a self-cogenerator, then $M / \operatorname{Rad}(M)$ is a $V$-module.

Proof. We note that $M / \operatorname{Rad}(M)$ is projective and a self-generator in $\operatorname{Mod}-R / J(R)$. By Proposition 3.2, $\operatorname{End}\left(M / \operatorname{Rad}(M)_{R}\right)$ is a right $V$-ring and, hence, $\operatorname{End}\left(M / \operatorname{Rad}(M)_{R / J(R)}\right)$ is a right $V$-ring. Thus, by Hirano [7, Thm. 3.11], $M / \operatorname{Rad}(M)_{R / J(R)}$ is a $V$-module and so $M / \operatorname{Rad}(M)_{R}$ is a $V$-module.

ACKNOWLEDGEMENT. The author would like to express his indebtedness and gratitude to the referee for his helpful suggestions and valuable comments.

\section{REFERENCES}

[1] T. Albu and C. Năstăsescu, Relative finiteness in module theory, Monographs and Textbooks in Pure and Applied Mathematics, vol. 84, Marcel Dekker, Inc., New York, 1984. MR 85k:16001. Zbl 556.16001.

[2] F. W. Anderson and K. R. Fuller, Rings and categories of modules, Graduate Texts in Mathematics, vol. 13, Springer-Verlag, New York, Heidelberg, 1974. MR 545281. Zbl 301.16001.

[3] C. Faith, Injective modules and injective quotient rings, Lecture Notes in Pure and Applied Mathematics, vol. 72, Marcel Dekker, Inc., New York, 1982. MR 83d:16023. Zbl 484.16009.

[4] C. Faith and P. Menal, A counter example to a conjecture of Johns, Proc. Amer. Math. Soc. 116 (1992), no. 1, 21-26. MR 92k:16033. Zbl 762.16011. 
[5] - The structure of Johns rings, Proc. Amer. Math. Soc. 120 (1994), no. 4, 1071-1081. MR 94j:16036. Zbl 803.16017.

[6] _ A new duality theorem for semisimple modules and characterization of Villamayor rings, Proc. Amer. Math. Soc. 123 (1995), no. 6, 1635-1637. MR 95g:16009. Zbl 834.16005.

[7] Y. Hirano, Regular modules and V-modules, Hiroshima Math. J. 11 (1981), no. 1, 125-142. MR 83f:16032a. Zbl 459.16009.

[8] Y. Kurata and K. Hashimoto, On dual-bimodules, Tsukuba J. Math. 16 (1992), no. 1, 85-105. MR 93i:16004. Zbl 792.16008.

[9] R. Wisbauer, Foundations of module and ring theory, revised and updated english ed., Algebra, Logic and Applications, vol. 3, Gordon and Breach Science Publishers, Philadelphia, PA, 1991, A handbook for study and research. MR 92i:16001. Zbl 746.16001.

TSUda: Department of Mathematics, Graduate School of Natural Science AND TeChNOLOGY, OKAYAMA UNIVERSITY, OKAYAMA 700, JAPAN

E-mail address: kentaro@math.okayama-u.ac.jp 


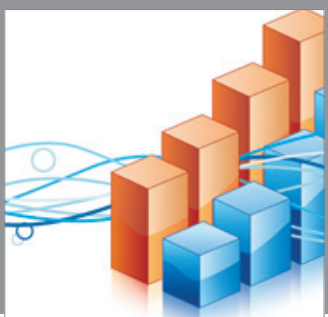

Advances in

Operations Research

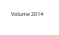

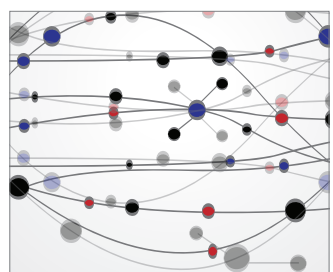

\section{The Scientific} World Journal
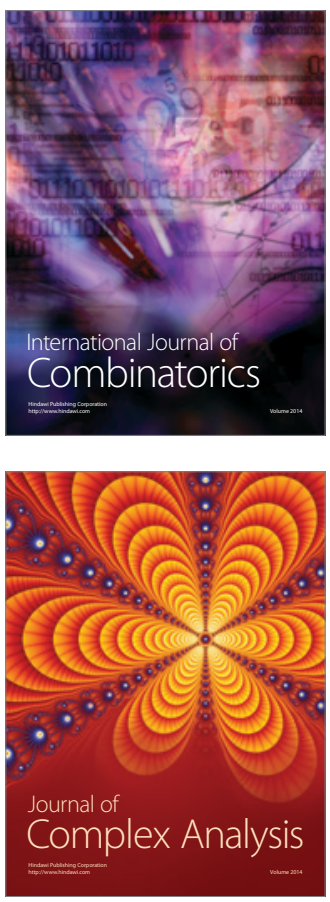

International Journal of

Mathematics and

Mathematical

Sciences
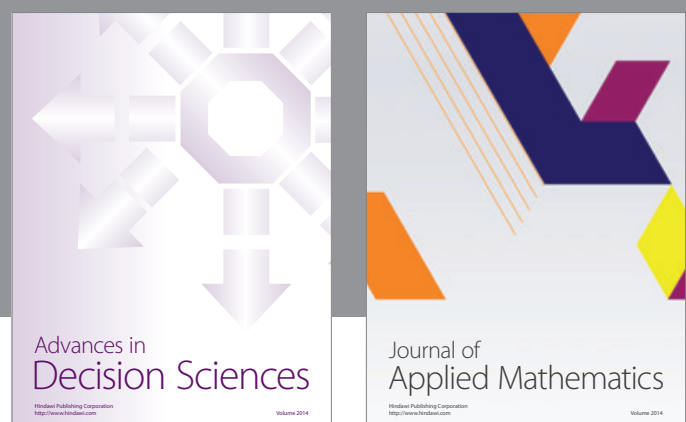

Journal of

Applied Mathematics
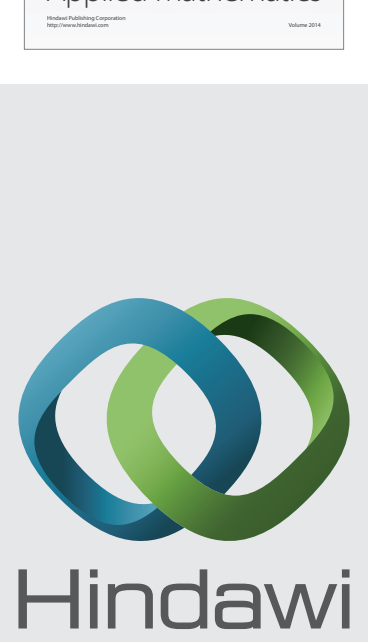

Submit your manuscripts at http://www.hindawi.com
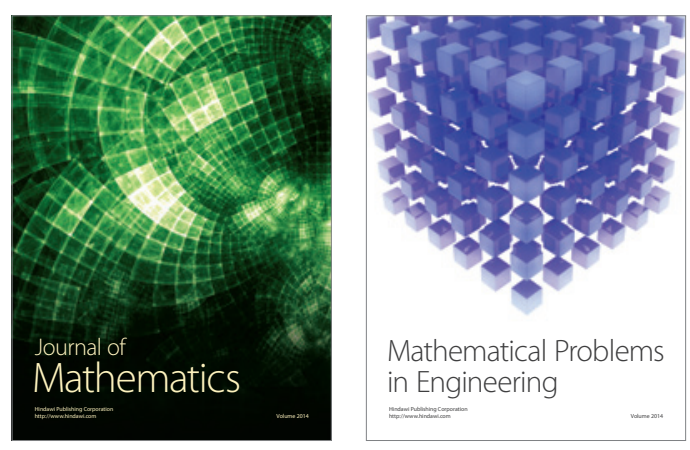

Mathematical Problems in Engineering
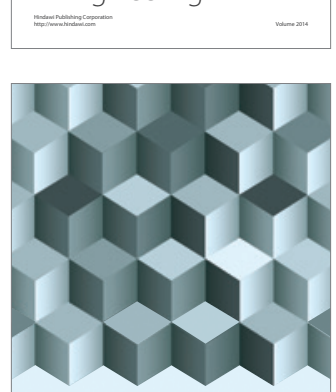

Journal of

Function Spaces
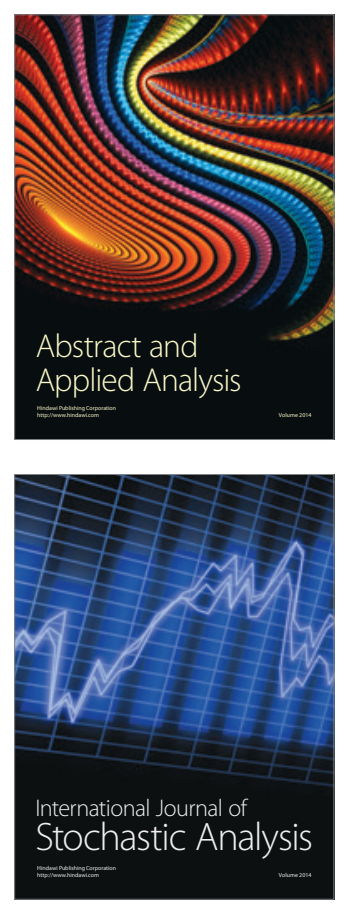

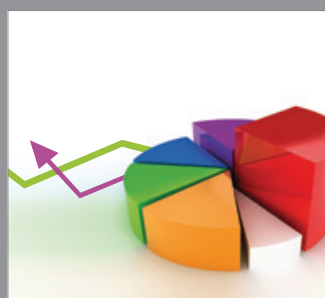

ournal of

Probability and Statistics

Promensencen
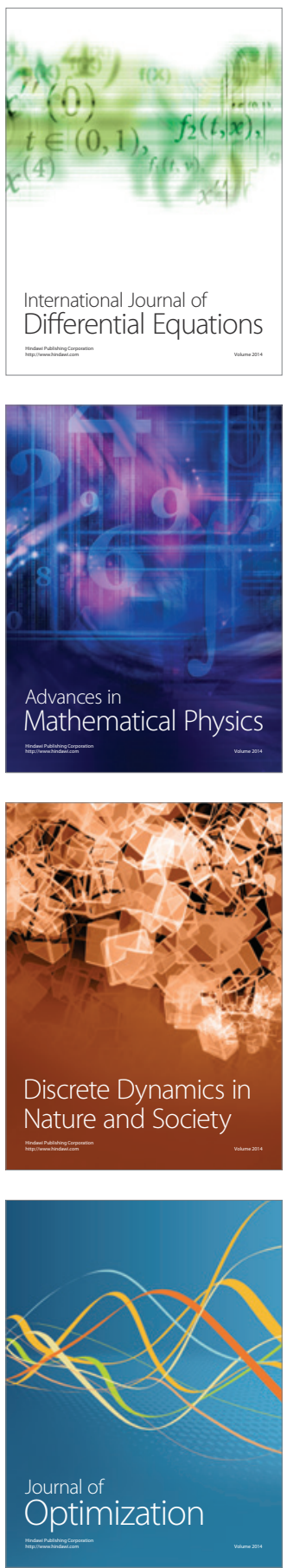